\title{
GaN HOMOEPITAXY FOR DEVICE APPLICATIONS
}

\author{
M. Kamp*, C. Kirchner*, V. Schwegler*, A. Pelzmann*, K.J. Ebeling*, \\ M. Leszczynski**, I. Grzegory**, T. Suski**, S. Porowski** \\ * Dept. of Optoelectronics, University of Ulm, 89069 Ulm, GERMANY, \\ Tel.: +49-731-5026050, Fax: +49-731-5026049, e-mail: markus.kamp@e-technik.uni-ulm.de \\ ** High Pressure Research Center, Warsaw, POLAND
}

Cite this article as: MRS Internet J. Nitride Semicond. Res. 4S1, G10.2(1999)

\section{$\underline{\text { Abstract }}$}

Epitaxial growth on GaN single bulk crystals sets new standards in GaN material quality. The outstanding properties provide new insights into fundamental material parameters (e.g. lattice constants, exciton binding energies, etc.) being not accessible by heteroepitaxial growth on sapphire or SiC. With MOVPE and MBE we realized unstrained GaN layers with dislocation densities about six orders of magnitude lower than in heteroepitaxy. Those layers revealed an exceptional optical quality as determined by a reduction of the photoluminescence linewidth from 5 to $0.1 \mathrm{meV}$ and a reduced XRD rocking curve width from 400 to 20 arcsec.

Only recently, progress in surface preparation allowed morphologies of the layers suitable for device applications. We report on InGaN/GaN MQW structures as well as the first $\mathrm{GaN} p n$ and $\mathrm{InGaN} / \mathrm{GaN}$ double heterostructure LEDs on $\mathrm{GaN}$ single bulk crystals. Those LEDs are twice as bright as their counterparts grown on sapphire. In addition they reveal an improved high power characteristics, which is attributed to an enhanced crystal quality and an increased $p$-doping. Time resolved electroluminescence measurements proof that band/band recombination is the dominant emission mechanism for the InGaN/GaN LEDs.

\section{Introduction}

Due to its excellent optical and electrical properties, $\mathrm{GaN}$ attracts worldwide attention for devices and fundamental research. The wide direct bandgap, the high luminescence efficiency and the thermal, mechanical, and chemical robustness make group III-nitride semiconductors the superior material system for optoelectronic devices in the UV to visible range. Despite exceptional progress, group III-nitrides technology still suffers from mismatched heteroepitaxial growth. Mismatch in lattice constants and thermal expansion coefficients between substrate (mostly sapphire or $\mathrm{SiC}$ ) and epitaxial layer inhibit perfect crystal formation, resulting in $10^{9}$ to $10^{10}$ threading dislocations per $\mathrm{cm}^{2}$.

Homoepitaxial growth of $\mathrm{GaN}$ has proven its tremendous potential to achieve superior material quality resulting in extremely narrow photoluminescence (PL) linewidths [1],[2] and a reduction of the dislocation densities by six orders of magnitude. These material qualities can only be attained using a substrate which is identical in crystal structure, lattice parameter and thermal expansion coefficient. Under those conditions, two-dimensional layer-by-layer growth can be obtained and the generation of dislocations can be inhibited. Additional process steps such as nitridation and nucleation layers, mandatory in heteroepitaxy of $\mathrm{GaN}$, are no longer required, thus significantly simplifying the growth process. Besides the fundamental advantages of homoepitaxy, GaN substrates have a high thermal conductivity facilitating high power applications. Since they are electrically conductive, too, they provide additional freedom for the device design (e.g. vertical current transport) and simplify the device processing. Beside above mentioned advantages, $\mathrm{GaN}$ substrates are of particular interest for GaN-based blue laser, since 
simple facet cleaving [3] becomes feasible and the far field pattern will be improved due to the relative high index of refraction of the GaN substrates. These outstanding properties justify the enormous endeavor conducted at several laboratories on the growth of single crystal GaN substrates [4],[5],[6]. Even when the effort for those substrates is probably too high for mass products such as LEDs, it might be worth for high power laser applications.

\section{$\underline{\text { Experimental }}$}

Substrate preparation

The GaN substrates have been grown in a high-temperature high-pressure process where the $\mathrm{GaN}$ is formed at $\mathrm{N}_{2}$ pressures up to $20 \mathrm{kbar}$ and at temperatures up to $1600^{\circ} \mathrm{C}$ from atomic nitrogen dissolved in a Ga melt. At a growth rate of approximately $100 \mu \mathrm{m} / \mathrm{h}$ perpendicular to the c-plane, the wurtzite crystals are grown up to areas of some $100 \mathrm{~mm}^{2}$ at a thickness of about $200 \mu \mathrm{m}$. Undoped crystals used in this study reveal an flat (000-1) surface (i.e. N-polarity) and a rough (0001) surface (i.e. Ga-polarity), following E. Hellman's convention on the orientation [7]. The (000-1) surface can be mechano-chemically polished to achieve an atomically flat surface. The initial roughness of the (0001) side requires a polishing for device quality growth. Due to the chemically inertness of the (0001) surface this can only be achieved by mechanical polishing.

The crystal quality of the GaN substrates is excellent as indicated by x-ray rocking curve measurements [8]. Using $\mathrm{Cu} K \alpha_{1}$ radiation, linewidths of 20 arcsec are obtained for the (0002) reflex. The excellent structural properties are also pointed out by very low dislocation densities ranging from $10^{3}-10^{5} \mathrm{~cm}^{-2}$. The optical quality however is poor, near-bandgap excitonic transitions are not visible, weak PL at $380 \mathrm{~nm}$ and at $530 \mathrm{~nm}$ is observed at room temperature (RT).

\section{Epitaxial growth}

The homoepitaxial growth of GaN is performed in a horizontal, RF heated, water cooled quartz MOVPE reactor (AIXTRON AIX $200 \mathrm{RF}$ ) operated at low pressures. Trimethylgallium (TMGa), Trimethylindium (TMIn), Trimethylaluminum (TMAl), ammonia $\left(\mathrm{NH}_{3}\right)$, silan $\left(\mathrm{SiH}_{4}\right)$ and bis-cyclopentadienylmagnesium $\left(\mathrm{Cp}_{2} \mathrm{Mg}\right)$ are used as precursors, hydrogen is used as carrier gas. The one side polished $\mathrm{GaN}$ substrates (approximately $8 \times 6 \mathrm{~mm}^{2}$ in size) were heated to $1060^{\circ} \mathrm{C}$ under a steady flow of ammonia to avoid surface degradation. During growth, the flow rates of $\mathrm{NH}_{3}$ and $\mathrm{TMGa}$ were kept at $2.0 \mathrm{slm}$ and $17 \mu \mathrm{mol} / \mathrm{min}$, respectively. The $\mathrm{Cp}_{2} \mathrm{Mg}$ flow rate was $90 \mathrm{nmol} / \mathrm{min}$ for $p$-type doping. After growth, thermal annealing under nitrogen ambient at $750^{\circ} \mathrm{C}$ was performed for 5 min to obtain $p$-type conductivity.

\section{Characterization}

Photoluminescence measurements at RT and $4 \mathrm{~K}$ are performed using a HeCd laser $(\lambda=325 \mathrm{~nm})$ with an excitation density of approx. $10 \mathrm{~mW} / \mathrm{mm}^{2}$. X-ray diffraction (XRD) with $\mathrm{CuK} \alpha_{1}$ radiation is used to characterize the structural quality of the binary layers as well as the composition of the ternary ones. Hall measurements of the epitaxial layers are inhibited by the highly conductive GaN substrate.

\section{Devices}

LEDs are fabricated taking advantage of the vertical device structure. Ni/Au contacts $(50 / 200 \mathrm{~nm})$ are evaporated as $p$-contacts. Dicing is achieved by simple cleaving, since layer and substrate are identical in their crystal structure and orientation. Subsequently, the devices are mounted on a copper plate using silver glue as backside contact material ( $n$-type). The LEDs are characterized by $I-V$ measurements, their output power, and their electroluminescence spectra under cw- as well as ns-short-pulse-excitation. 


\section{$\underline{\text { Results and Discussion }}$}

Surface orientation and preparation

Both orientations (N-polarity (000-1) and Ga-polarity (0001)) of undoped single crystal substrates have been investigated in this study. Figure 1 compares the photoluminescence of $\mathrm{GaN}$ layers grown under identical conditions on $\mathrm{GaN}$ substrates of both orientations.

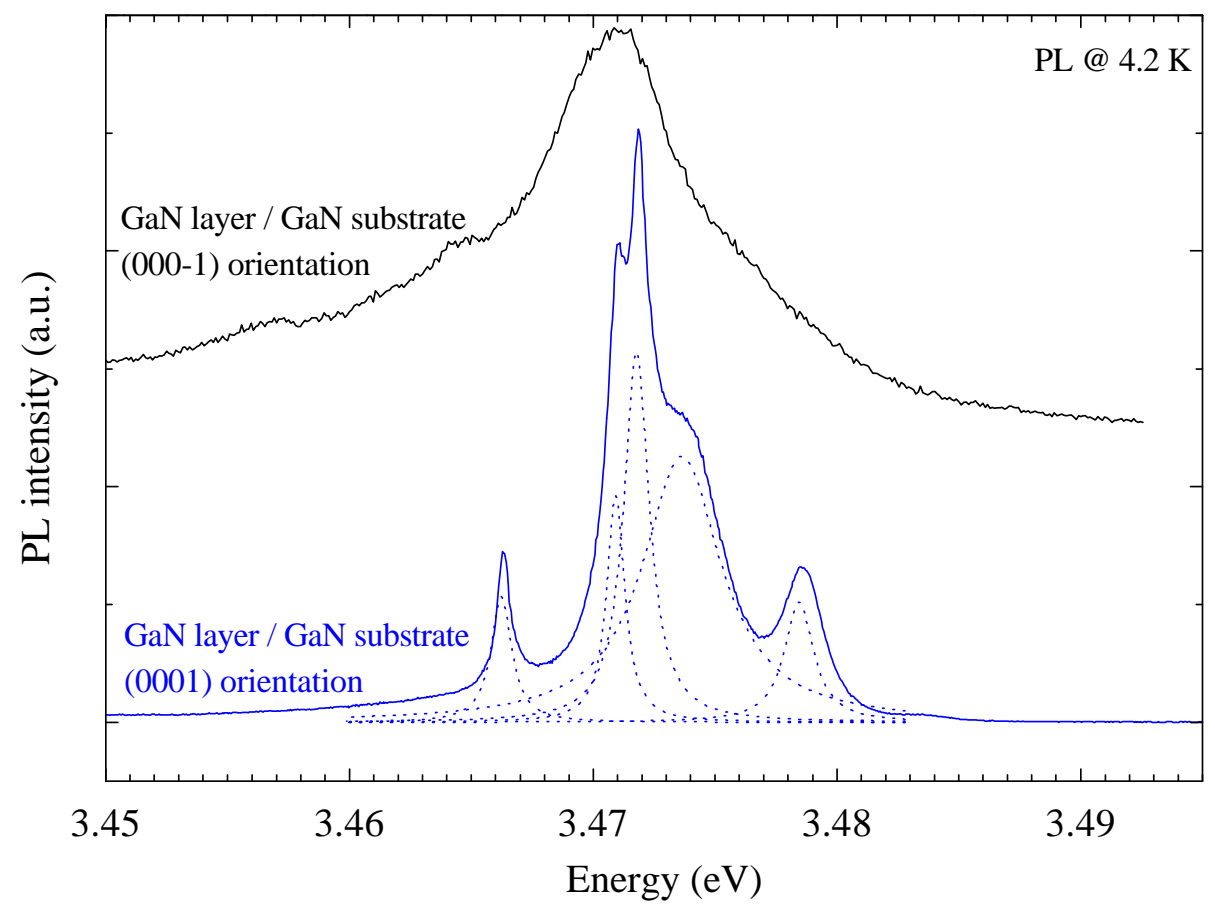

Fig 1.: Photoluminescence of epitaxial GaN layers (MBE) on (000-1)- and (0001)-oriented GaN substrates. Linewidth is as low as $0.5 \mathrm{meV}$ for the (0001)-orientation.

At a PL linewidth of approx. $2 \mathrm{meV}$ the material quality of the epitaxial layer grown on (000-1) oriented substrates compares favorably to heteroepitaxial growth. However, the properties of epitaxial films deposited on (0001) oriented substrates are clearly superior. Due to the outstanding material quality achieved on the (0001) oriented substrates $(0.5 \mathrm{meV}$ for the bound excitons in this MBE grown layer), this orientation has been used for the subsequently described work. The differences between both orientations can be traced back to the significantly different free surface energies of the orientations. From ab-initio calculations it is determined that the free surface energy of the (000-1) surface is significantly higher than the one of the (0001) surface [9]. From this point, the (0001) orientation provides a more stable surface with a lower probability of dopant incorporation [10].

For the following experiments the Ga-terminated side was mechanically polished to remove the initial roughness of the (0001) surface. This process, however, is known to create sub-surface damage which interferes with high quality epitaxial growth on such treated substrates. In conventional III-V technology, this damage is chemically removed, an approach not accessible for the nitrides, as there is no suitable etchant known. Therefore, chemically assisted ion beam etching (CAIBE), a dry etching technique where the physical and chemical 
components of the etching process can be controlled fairly independently, is used to remove residual sub-surface damage. For direct comparison one half of a sample is CAIBE treated, whereas the other part is left unetched. Therefore, the substrate surface is partially coated with photoresist, acting as an etch mask. Argon and chlorine gas are used as physical and chemical etching components, respectively. Within 10 minutes an etch depth of approx. $300 \mathrm{~nm}$ was achieved using an ion energy of $400 \mathrm{eV}$ which is reduced to $100 \mathrm{eV}$ towards the end to minimize the ion damage. Nevertheless, the process is supposed to create an ion damage up to a depth of $8 \AA$. Since the etch rate depends only weakly on the chlorine flux, the process is regarded to be dominated by the physical component, i.e. sputtering. Figure 2a shows a SEM micrograph of a homoepitaxial GaN layer grown on a substrate which has been treated as described above.
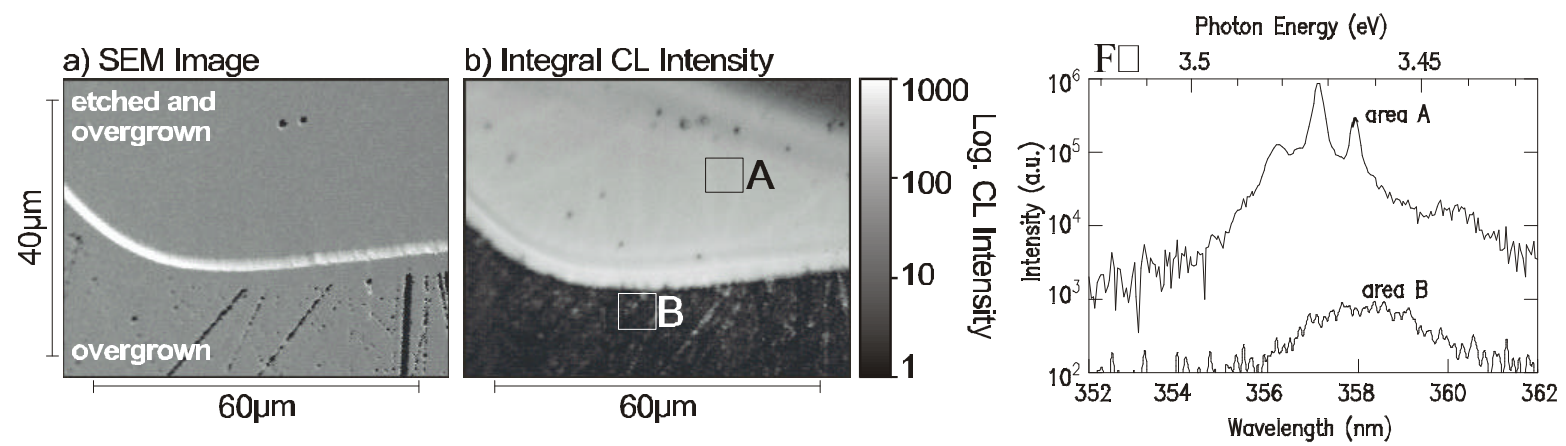

Fig 2.: SEM image (2a), corresponding cathodoluminescence (CL) intensities (2b) and local CL spectra (2c) obtained from an epitaxial GaN layer grown on partially CAIBE treated (0001)oriented GaN substrates. CL by F. Bertram, T. Riemann, and J. Christen (Univ. Magdeburg).

The micrograph depicts the surface of a MOVPE grown GaN film just at the borderline between the etched and overgrown part (upper part) and the overgrown part which was not previously etched (lower part). The CAIBE-etched part of the sample reveals a significantly improved surface topology with almost no visible scratches, trenches, or holes. Figure $2 \mathrm{~b}$ shows the corresponding CL intensity distribution of the same region of the sample. On the etched part, the intensity variation is almost negligible. In contrast, the area being not etched yields only weak CL signals (1000 times less in intensity) which also fluctuate locally. The comparison of the local CL spectra reveals large differences. The measured linewidth of the spectrum taken from the etched region (fig. $2 \mathrm{a}$, area $\mathrm{A}$ ) is ten times narrower than that of the non-etched region (fig. 2a, area B). However, the narrow CL spectra obtained from area A are still clearly resolution limited by the employed setup. The following mechanism is proposed to be responsible for the observations [11]. After mechanical polishing, the bulk GaN substrates reveal a smooth and mirror-like surface, however, sub-surface damage is induced by the polishing. By heating the substrates to growth temperature under ammonia during the MOVPE process, the damage becomes obvious revealing the observed trenches and scratches in the surface (fig. $2 \mathrm{a}$, lower part). This experimental finding can be understood by dissociation and subsequent desorption of the damaged GaN. Eventually, during the heat up phase of the MOVPE growth, the substrate temperature of about $1100^{\circ} \mathrm{C}$ becomes high enough to achieve chemical etching (dissociation) under $\mathrm{NH}_{3}$ even of non-deteriorated GaN layers [12]. Whenever CAIBE is applied before annealing, the originally mirrorlike surface morphology is kept mirrorlike during the mostly physical etching process and thereby transferred into the undisturbed part of the substrate. However, ion-beam induced damage is created by the CAIBE process. This damage, nevertheless, is distributed homogeneously over the sample surface. During annealing in the MOVPE system this damage will dissociate revealing an almost perfect and smooth surface. 
Excess metallic Ga left on the surface is desorpt due to the exceptional high growth temperature of the group III-nitrides.

The necessity of the CAIBE treatment for devices based on single crystal substrates can best be seen in Fig. 3 showing evaporated Ni/Au metal contacts on a GaN pn-junction LED in an area without (Fig. 3a) and with prior CAIBE treatment (Fig 3b). The region of the device which was not CAIBE-treated reveals deep trenches as described earlier. Since the depths of the trenches is above the typical thickness of the $p$-GaN layer any metallization covering the sidewalls of the trenches will short circuit the $p n$-junction of the device.
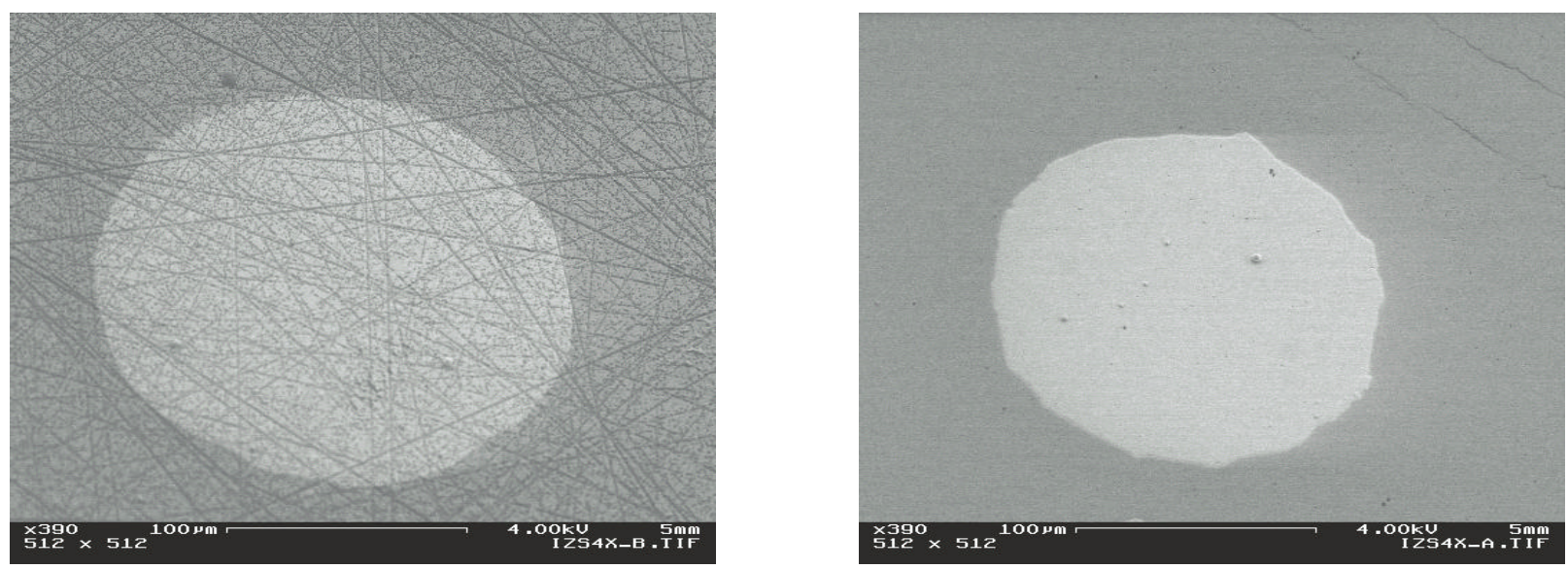

Fig. 3: SEM micrograph of the surface of a homoepitaxial GaN pn-junction LED with evaporated $\mathrm{Ni} / \mathrm{Au}$ metal contacts, without (3a) and with (3b) CAIBE treatment prior to growth (different areas of the same sample). 
Binary and ternary layer properties

Homoepitaxial GaN layers with outstanding properties have been achieved using above described CAIBE technique (Fig. 4). PL at 4.2 K reveals free excitons A, B, C as well as excited states of those excitons, where the identification is verified by reflectance measurements included in the figure. The linewidth of the bound excitons $(3.464-3.474 \mathrm{eV})$ is as low as $0.1 \mathrm{meV}$.

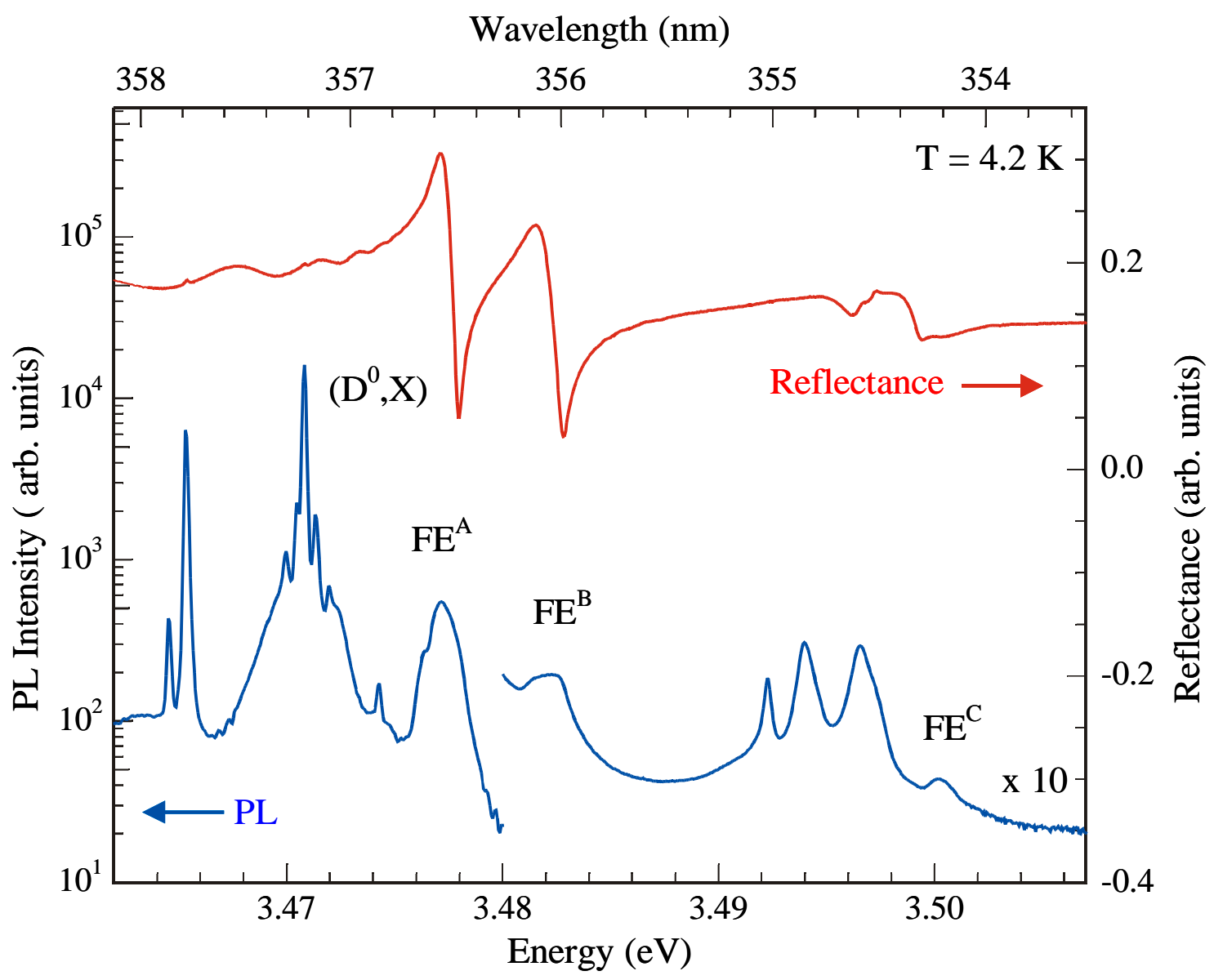

Fig. 4: High resolution photoluminescence (log. scale) and reflectance (lin. scale) of a $2 \mu \mathrm{m}$ epitaxial GaN layer grown on CAIBE treated (0001)-oriented GaN substrates. Measurements by

K. Kornitzer, K. Thonke, and R. Sauer (Univ. Ulm, Dept. of Semiconductor Physics).

The structural quality of the homoepitaxial GaN layers is extremely high as indicated by the narrow linewidth of approx. 20 arcsec ((0002) reflex), revealing the low dislocation density and the negligible mosaicity of the GaN layers grown on single crystal GaN substrates. A minor lattice mismatch of approx. a/a $10^{-4}$ between layer and substrate is due to the high carrier concentration of the substrates [8]. The outstanding xray data confirm that homoepitaxial growth conserves or even improves the excellent structural quality and low dislocation density of the GaN substrates, as expected. 
Ternary III-N compounds have been investigated as building blocks for doubleheterostructure- (DH) and MQW-LEDs. The PL of $50 \mathrm{~nm}$ thick InGaN layers is shown in Fig 5. For comparison the PL of a heteroepitaxially layer grown under identical conditions (but employing a nucleation layer) is included. The observed shift in the emission wavelength can be understood by a different surface temperature between the highly heat conductive and partially absorbing GaN substrate and the sapphire. This causes a higher surface temperature for the GaN substrates at a nominally identical susceptor temperature, leading to a reduced In incorporation and the observed slightly lower emission wavelength. Fig 6. reveals a analogously comparison for $\mathrm{InGaN} / \mathrm{GaN}(3 \mathrm{~nm} / 3 \mathrm{~nm})$ multiple quantum well structures.

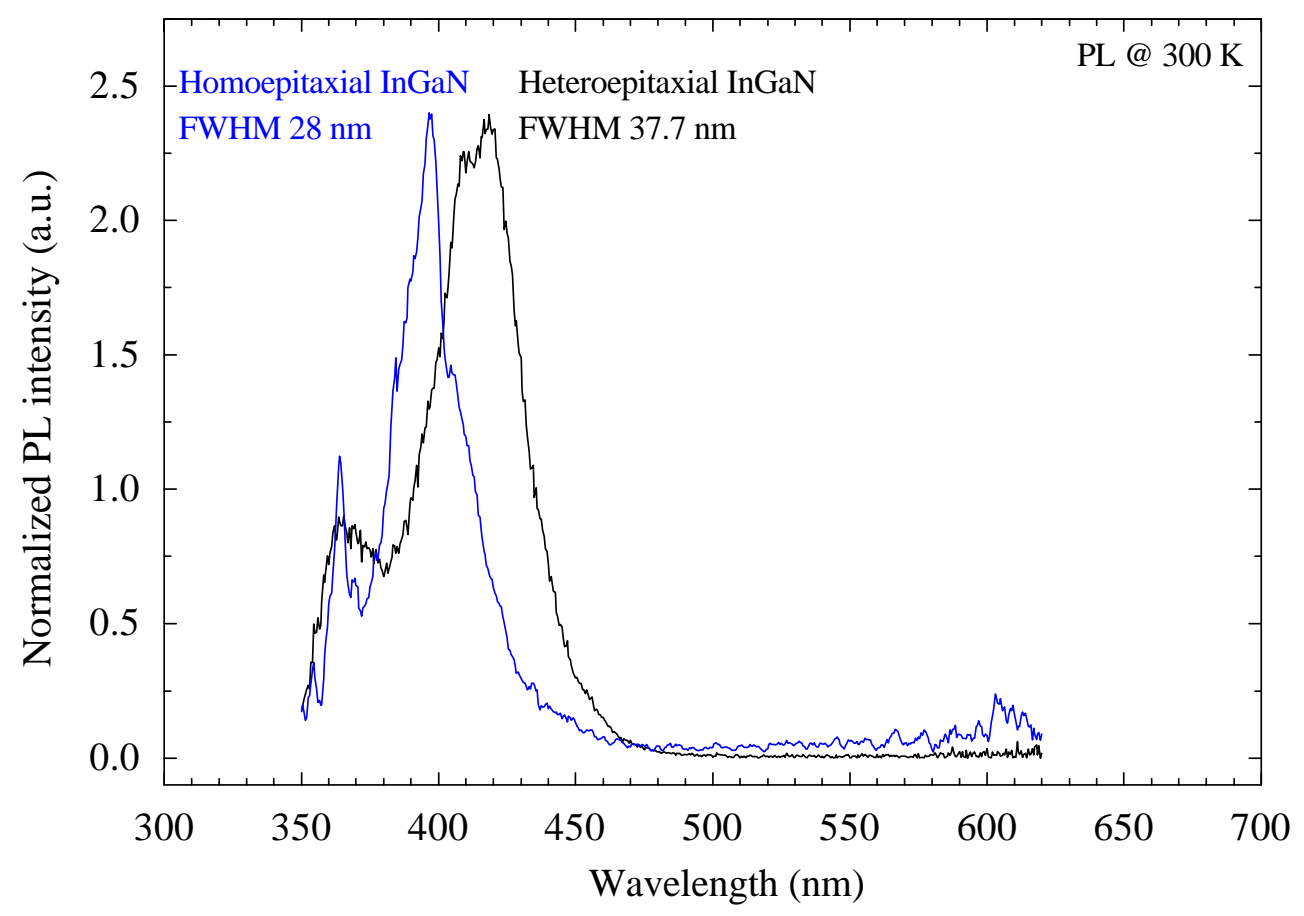

Fig. 5: Room temperature photoluminescence of approx. $50 \mathrm{~nm}$ InGaN layers grown on GaN- and sapphire-substrates, respectively. 


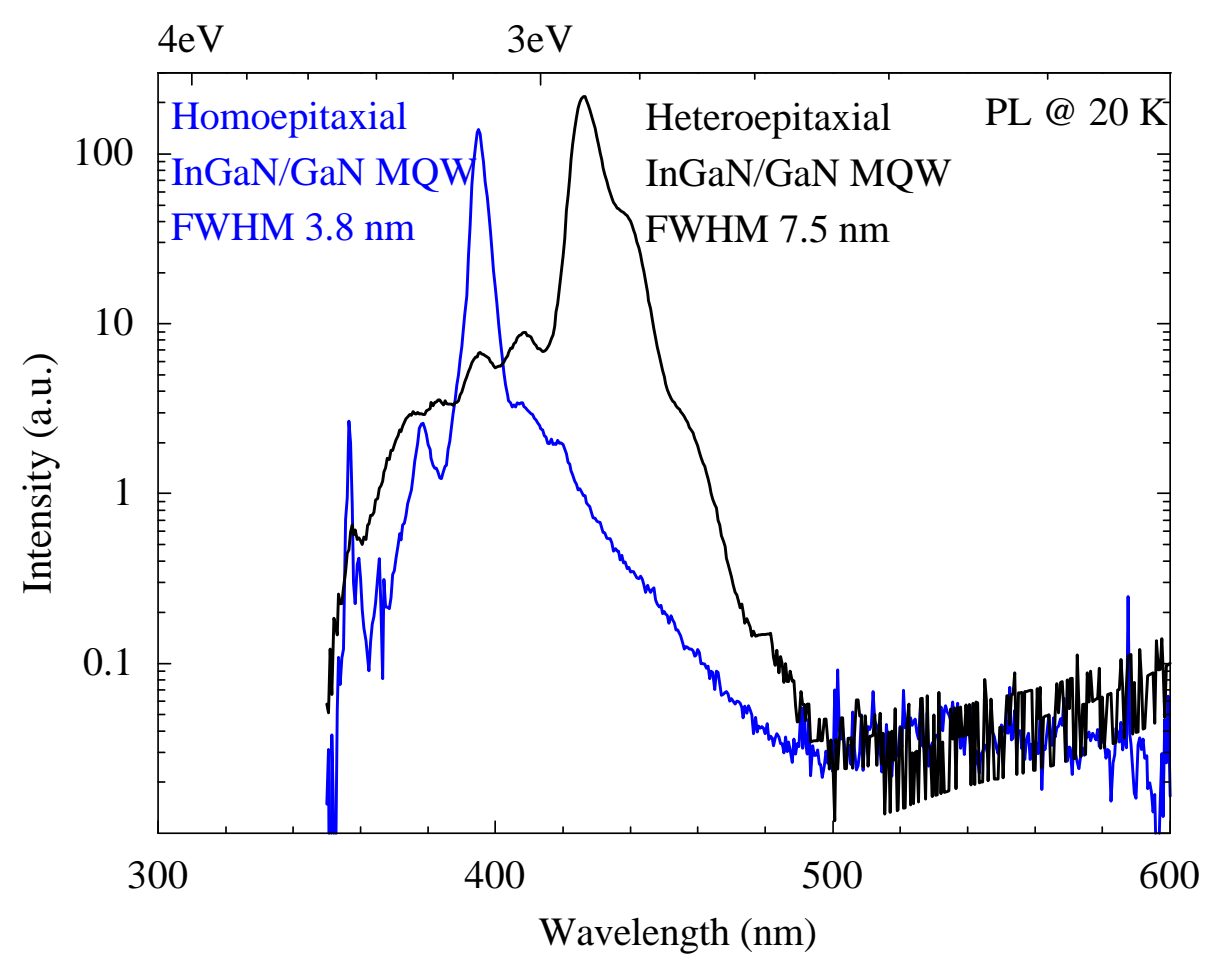

Fig. 6: Photoluminescence of $\mathrm{InGaN} / \mathrm{GaN}(3 \mathrm{~nm} / 3 \mathrm{~nm})$ multiple quantum well structures grown on $\mathrm{GaN}$ - and sapphire-substrates, respectively $(20 \mathrm{~K})$.

Light emitting diodes

Initial LED structures have been homotype $p n$-junction LEDs. Only the regions of the device which underwent a CAIBE treatment yield functional devices, as becomes obvious from Fig. 3. The EL of those devices is depicted in Fig. 7 for various current densities. The LEDs show an intense, single peak emission at about $420 \mathrm{~nm}$ wavelength with a linewidth of $60 \mathrm{~nm}$ for low currents. UV emission is not observed even under the highest current densities. It is remarkable that the emission wavelength is constant at approx. $425 \mathrm{~nm}$ up to current densities of $3000 \mathrm{kA} / \mathrm{cm}^{2}$. As was initially pointed out by S.Nakamura et al. for GaN $p n$-junction LEDs this is a clear indicative of the high quality of the $p$-type material obtainable by homoepitaxial growth [13]. With increasing free hole concentration, the PL transition at $425 \mathrm{~nm}$ is found to increase in intensity [14],[15]. Only a very high density of those recombination centers can guarantee an emission at $425 \mathrm{~nm}$ in EL even under high current densities. Once the injected carriers outnumber these recombination centers the surplus carriers recombine under emission of UV light at about $380 \mathrm{~nm}$ [16]. The EL obtained from heteroepitaxial LEDs grown on sapphire under identical conditions is also included for comparison (dashed line). The heteroepitaxial devices reveal a clear shift towards shorter wavelengths which is attributed to an inferior quality of the $p$-material at the $p n$-junction. Furthermore, the homoepitaxial LED is approx. twice as bright as their counterpart on sapphire. 


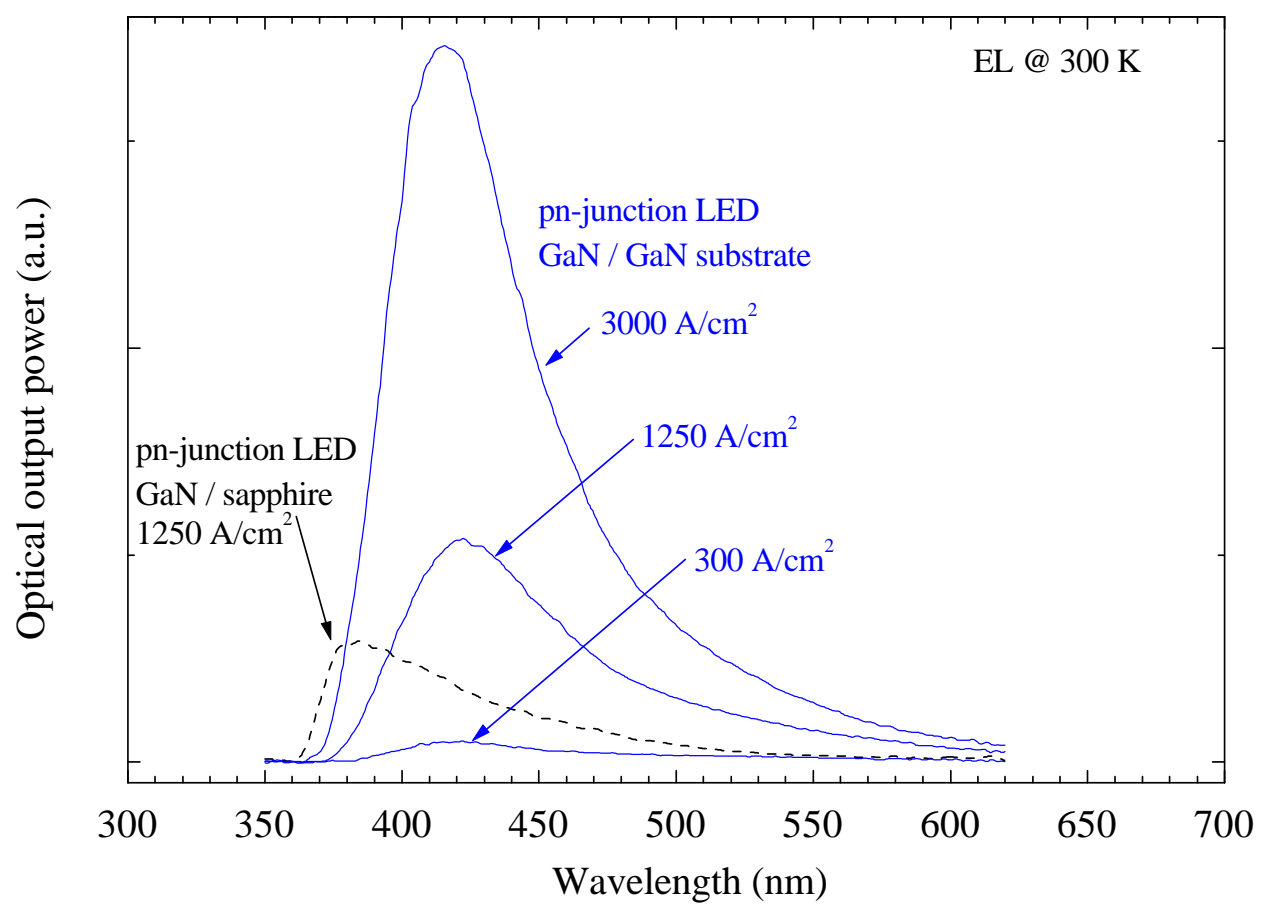

Fig. 7: Electroluminescence (EL) of a GaN homojunction $p n$-LED grown on GaN substrate. Emission spectra at various current densities. At a given current density, the homoepitaxial devices are twice as bright as comparable LEDs grown on sapphire (dashed line).

In addition to above homojunction LEDs, first InGaN/GaN DH-LEDs have been fabricated using single bulk crystal substrates. The EL of those devices is depicted in Fig. 8. Even at low current densities of $6 \mathrm{~A} / \mathrm{cm}^{2}$ decent EL is observed revealing a significant lower density of non-radiative recombination centers than in their on-sapphire-grown counterparts. Time resolved EL is measured to reveal the recombination mechanisms (Fig. 9) [17]. A single exponential decay with a time constant as low as $1 \mathrm{~ns}$ clearly identifies band/band recombination to be the dominant emission mechanism, since defect- as well as DAP-related electroluminescence transitions, as localized states, reveal a two order of magnitudes longer decay time (about $100 \mathrm{~ns}$ ) [18]. 


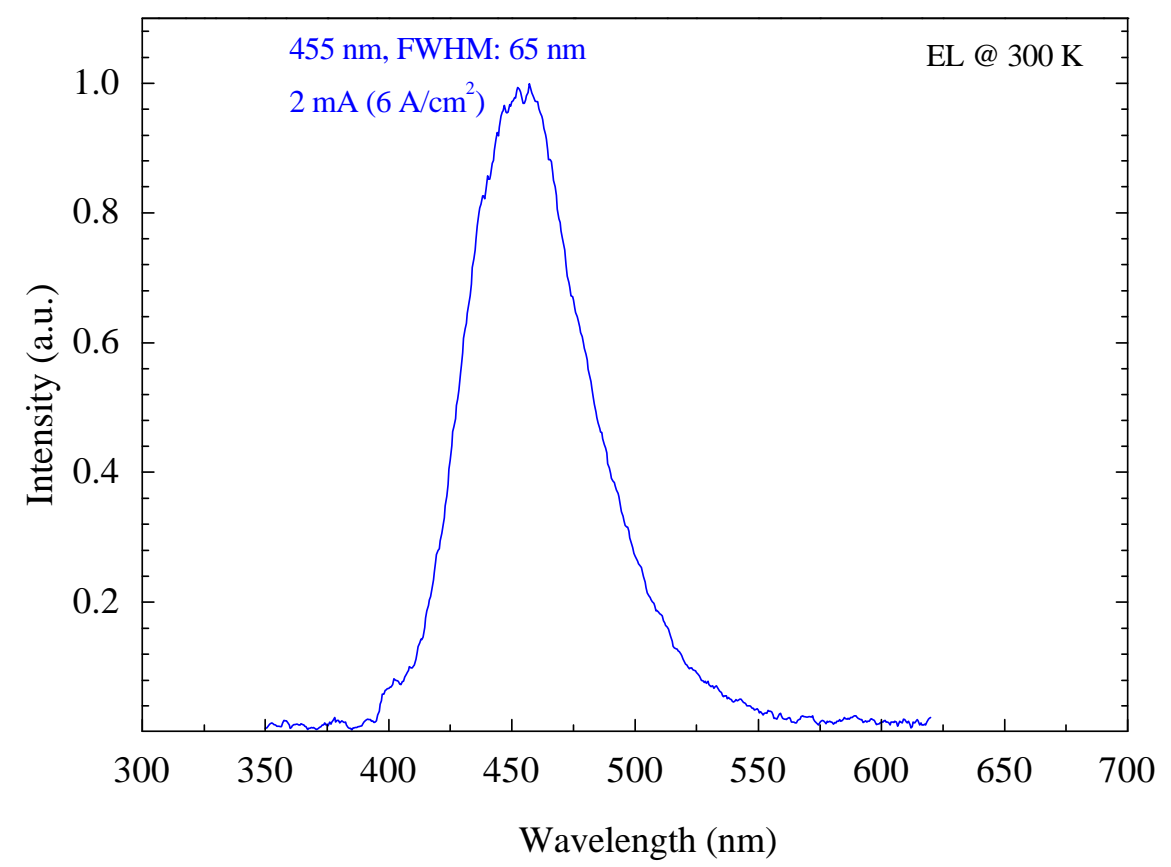

Fig. 8: Electroluminescence (EL) of a InGaN/GaN DH-LED grown on GaN substrate. Excellent EL is achieved at low current densities, revealing a low density of non-radiative defects.

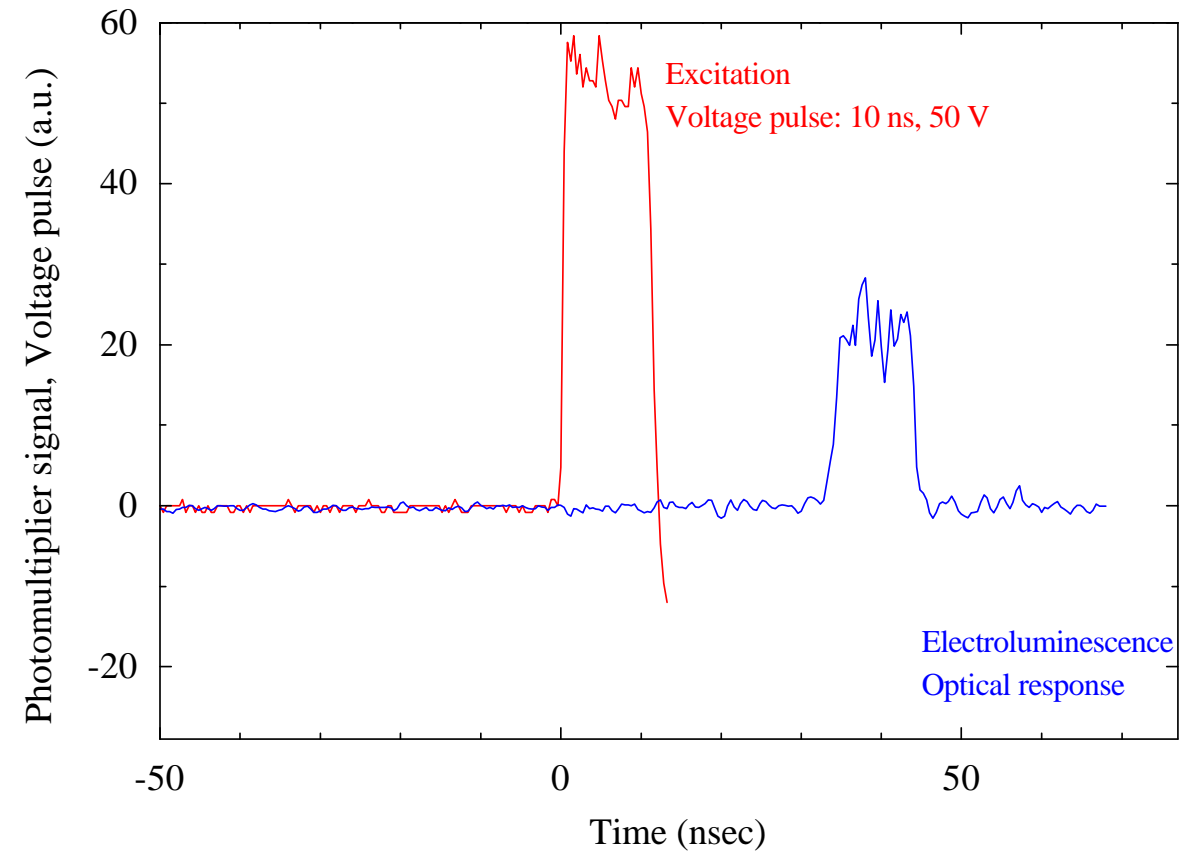

Fig. 9: Time resolved electroluminescence (EL) of a InGaN/GaN DH-LED grown on $\mathrm{GaN}$ substrate. Depicted is the EL after short-pulse-excitation at $50 \mathrm{~V}$ for $10 \mathrm{~ns}$ (pulse being also depicted). Exponential decay time is as short as $1 \mathrm{~ns}$ indicating band/band recombination. The pulse-signal delay of $35 \mathrm{~ns}$ is caused by the experimental setup. 
The current through the LED as depicted in Fig. 10 is obviously limited by the series resistance (i.e. contact and sheet resistances) of the devices, which can be deduced to about $1 \mathrm{k} \Omega$ from the currents at high voltages $\left(U_{D}\right.$ larger than a few $\left.\left(n_{B} T\right) / q\right)$. The voltage drop at the series resistance is most likely caused by the imperfect housing of the LEDs, since the devices have just been glued to a metal base using silver glue. The current then is then passed through the 200 $-300 \mu \mathrm{m}$ thick substrate of the vertical device. Further improvements are expected using a more elaborated contact and housing technology.

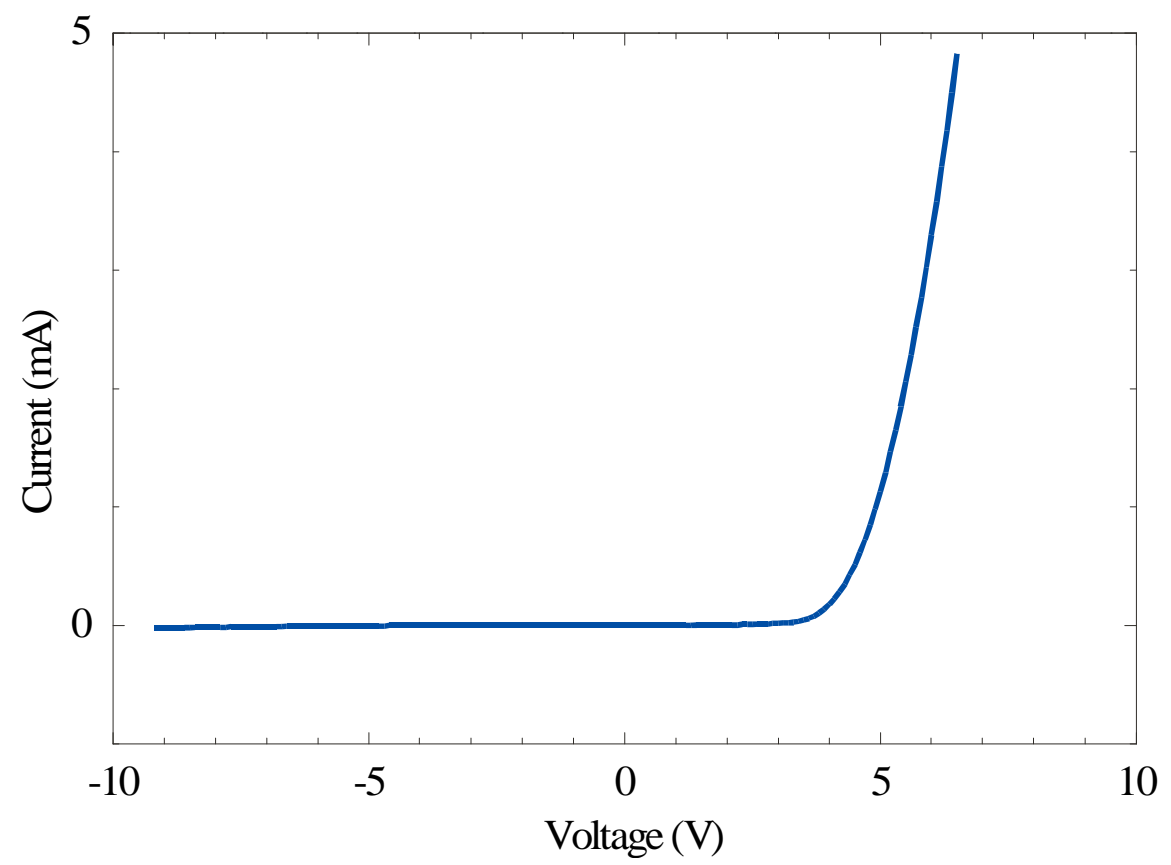

Fig. 10: Current/voltage characteristics of a GaN InGaN/GaN DH-LED. The curve reveals a good breakdown behavior and turn-on-voltage but still a rather high series resistance. The later is probably originated by contributions from the silver glue ( $n$-contact metal) and the $200-300 \mu \mathrm{m}$ thick $n$-layer (i.e. mostly the substrate).

\section{$\underline{\text { Summary }}$}

After introduction of a CAIBE dry-etching step GaN layers with outstanding optical qualities and excellent morphologies have been achieved on bulk GaN single crystal substrates. Thick InGaN layers as well as InGaN/GaN MQWs are clearly superior to their counterparts grown on sapphire. Homoepitaxial GaN LEDs reveal single-peak blue emission at $420 \mathrm{~nm}$, with a linewidth of $60 \mathrm{~nm}$. The excellent quality of the homoepitaxial LEDs grown on GaN substrates is revealed by a comparison with heteroepitaxially LEDs on sapphire. The homoepitaxial devices are twice as bright as the LEDs grown on sapphire. This and the wavelength shift of the LED on sapphire are indications for a higher hole concentration of the homoepitaxial device. First InGaN/GaN DH-LEDs have been fabricated and reveal excellent electroluminescence at $455 \mathrm{~nm}$ even at low current densities, indicating a low density of non-radiative defects. Time resolved EL measurements performed on those devices clearly evince band/band recombination to be the dominant mechanism in those devices. 


\section{Acknowledgments}

Several researchers severely contributed to this work, including M. Schauler, F. Eberhard, S. Menzel, and P. Unger from Dept. of Optoelectronics, F. Bertram, T. Riemann, and J. Christen from Univ. Magdeburg (CL measurements), K. Kornitzer, K. Thonke, and R. Sauer from Univ. Ulm, Dept. of Semiconductor Physics (high resolution PL measurements and reflectance). The authors gratefully acknowledge their valuable contributions as well as financial support by the German Federal Ministry of Education, Science, Research and Technology (BMBF) under contract no. 01 BM 419 / 9 and the Volkswagen Foundation.

\section{References}

[1] M. Mayer, A. Pelzmann, M. Kamp, K.J. Ebeling, H. Teisseyre, G. Nowak, M. Leszczynski, I. Grzegory, M. Bockowski, S. Krukowski, S. Porowski, G. Karczewski, Jpn. J. Appl. Phys. Part 2, Vol. 36, L1634-L1636 (1997).

[2] J. Baranowski, S. Porowski, Proc. 23rd Int. Conf. on the Physics of Semiconductors, Berlin 1996, edited by M. Scheffler and R. Zimmermann (World Scientific, Singapore 1996) vol. 3, (1996).

[3] S. Nakamura, M. Senoh, S. Nagahama, N. Iwasa, T. Yamada, T. Matsushita, H. Kiyoku, Y. Sugimoto, T. Kozaki, H. Umemto, M. Sano, K. Chocho, Appl. Phys. Lett. 73, 832 (1998).

[4] J. Karpinski, J. Jun and S. Porowski: J. Crystal Growth 66, 1-10 (1984).

[5] T. Detchprohm, H. Amano, K. Hiramatsu and I. Akasaki: J. Crystal Growth 128, 384-390 (1993).

[6] S. Sakai, S. Kurai, K. Nishino, K. Wada, H. Sato and Y. Naoi: Mat. Res. Soc. Symp. Proc. 449, 15-22 (1997).

[7] E. Hellman, MRS Internet J. Nitride Semicond. Res. 3, 11(1998).

[8] M. Leszczynski, H. Teisseyre, T. Suski, I. Grzegory, M. Bockowski, J. Jun, S. Porowski, K. Pakula, J.M. Baranowski, C.T. Foxon, T.S. Cheng, Appl. Phys. Lett. 69 (1), 73-75 (1996).

[9] T. Zywietz, J. Neugebauer, M. Scheffler,J. Northrup, Chris G. Van de Walle, MRS Internet J. Nitride Semicond. Res. 3, 26(1998).

[10] M. Leszczynski, B.K. Meyer, unpublished.

[11] M. Schauler, F. Eberhard, C. Kirchner, V. Schwegler, A. Pelzmann, M. Kamp K.J. Ebeling, F. Bertram, T. Riemann, J. Christen, M. Leszczynski, I. Grzegory, T. Suski, S. Porowski, accepted for Appl. Phys. Lett.

[12] Yu. Makarov, Yu. Karpov, private communication.

[13] S. Nakamura, T. Mukai and M. Senoh: Jpn. J. Appl. Phys. 30 No. 12A, L1998-L2001 (1991).

[14] U. Kaufmann, M. Kunzer, M. Maier, H. Obloh, A. Ramakrishnan, B. Santic, P. Schlotter, Appl. Phys. Lett. 72 (11), 1326-1328 (1998).

[15] S. Haffouz, B. Beaumont, M. Leroux, M. Laugt, P. Lorenzini, P. Gibart, L.G. HubertPfalzgraf, MRS Internet J. Nitride Semicond. Res. 2, 37(1997).

[16]F. Calle, E. Monroy, F.J. Sánchez, E. Muñoz, B. Beaumont, S. Haffouz, M. Leroux, P. Gibart, MRS Internet J. Nitride Semicond. Res. 3, 24(1998).

[17] V. Schwegler, C. Kirchner, M. Kamp K.J. Ebeling, M. Leszczynski, I. Grzegory, S. Porowski, submitted to Appl. Phys. Lett.

[18]F.S. Choa, J.Y. Fan, P.L. Liu, J. Sipior, G. Rao, G.M. Carter, Y.J. Chen, Appl. Phys. Lett. 69 (24), 3668-3670 (1996). 